\title{
MRD-Negative Remission Induced in EP300-ZNF384 Positive B-ALL Patients by Tandem CD19/CD22 CAR T-Cell Therapy Bridging to Allogeneic Stem Cell Transplantation
}

\author{
Xin-Yue Zhang (D) ${ }^{1,2, *}$ \\ Hai-Ping Dai $\mathbb{D}^{1,2, *}$ \\ Ling Zhang ${ }^{1,2}$ \\ Si-Ning Liu ${ }^{1,2}$ \\ Yin Dai ${ }^{1,2}$ \\ De-Pei Wu ${ }^{1,2}$ \\ Xiao-Wen Tang ${ }^{1,2}$
}

'National Clinical Research Center for Hematologic Diseases, Jiangsu Institute of Hematology, The First Affiliated Hospital of Soochow University, Suzhou, 215006, People's Republic of China; ${ }^{2}$ Institute of Blood and Marrow Transplantation, Collaborative Innovation Center of Hematology, Soochow University, Suzhou, 215I23, People's Republic of China

*These authors contributed equally to this work

Correspondence: De-Pei Wu; Xiao-Wen Tang

Department of Hematology, The First Affiliated Hospital of Soochow University, Jiangsu Institute of Hematology, Suzhou,

People's Republic of China

$\mathrm{Tel}+8651267781856$

Fax +865I265II3556

Email drwudepei@163.com;

xwtang1020@163.com

\begin{abstract}
EP300-ZNF384-positive B cell acute lymphoblastic leukemia (B-ALL) patients are reported to have a unique immunophenotype with high expression of $\mathrm{CD} 19$ and $\mathrm{CD} 22$, weak expression of $\mathrm{CD} 20$ and aberrant expression of $\mathrm{CD} 13$ and/or CD33, sensitivity to chemotherapy and a favorable outcome. To date, the cases of only 53 patients have been reported, albeit few reports on salvage therapy when conventional chemotherapies failed. Here, we describe two relapsed and refractory adult B-ALL patients with EP300-ZNF384 who achieved second remission through tandem CD19/CD22 CAR T-cell therapy. Grade 3 and 2 cytokine release syndrome were observed in cases 1 and 2, respectively. No immune effector cell-associated neurotoxicity syndrome was detected. Both patients underwent consolidate haploidentical hematopoietic stem cell transplantation (HSCT), and each maintained measurable residual disease-negative remission for 14 and 13 months, respectively. Our study suggests that CD19/CD22 CAR T-cell therapy bridging to allogeneic HSCT may be a viable option for EP300-ZNF384-positive B-ALL.
\end{abstract}

Keywords: chimeric antigen receptor T-cells, CD19/CD22, EP300-ZNF384, acute lymphoblastic leukemia, relapsed/refractory

\section{Introduction}

B-cell acute lymphoblastic leukemia (B-ALL) represents a group of highly heterogeneous hematological malignancies. Approximately 55\% of B-ALL patients present with normal cytogenetics at diagnosis and are stratified into a standard risk group, many of whom, however, experienced relapse under conventional treatment strategies. ${ }^{1}$ With the application of whole-transcriptome sequencing, a growing number of cryptic aberrations have been revealed, which facilitate diagnosis, risk stratification, and targeted therapy in B-ALL patients with a normal karyotype.

EP300-ZNF384 is generated by a cryptic $\mathrm{t}(12 ; 22)(\mathrm{p} 13 ; \mathrm{q} 13)$ chromosome translocation and can be detected in 4.3-5.7\% of B-ALL patients. ${ }^{2,3}$ EP300-ZNF384positive B-ALL patients often have a high expression of CD19 and CD22, weak expression of CD20 and aberrant expression of CD13 and/or CD33. ${ }^{4}$ These patients also have a distinct gene signature with concurrent upregulation of the JAK-STAT pathway, reduced expression of B-cell regulators and poor DNA repair capacity. ${ }^{3}$ Based on small-scale retrospective studies, these patients are sensitive to chemotherapy and have a favorable clinical outcome. Because of its low incidence, 
there is a shortage of large-sample clinical studies focusing on this entity, and studies on salvage therapy for patients in whom conventional treatments fail are especially rare. ${ }^{5}$ Chimeric antigen receptor (CAR) T-cell therapy has shown an encouraging efficacy in refractory and relapsed $(\mathrm{R} / \mathrm{R})$ B-ALL. ${ }^{6}$ Here, we report the successful application of CAR T-cell therapy using a tandem CD19/CD22 CAR in two R/R B-ALL patients with the EP300-ZNF384 fusion. The schematic of the CD19/CD22 CAR structure is shown in Figure 1A.

\section{Case Reports}

Case 1 was a 30-year-old male diagnosed in February 2017 with B-ALL in the standard risk group. He received induction chemotherapy with daunorubicin, cyclophosphamide, vincristine, prednisone and L-asparaginase and achieved complete remission (CR), which he maintained under consolidation and maintenance chemotherapy for 3 consecutive years according to the GRAAPH-2003 protocol. $^{7}$ In April 2020, hematologic relapse was detected in a regular follow-up. BM aspirates showed $7 \%$ blasts, with negative peroxidase staining. Flow cytometry revealed that the blasts were positive for $\mathrm{CD} 13, \mathrm{CD} 19, \mathrm{CD} 22, \mathrm{CD} 33, \mathrm{CD} 34, \mathrm{CD} 38$, CD79a, HLA-DR and negative for CD20, consistent with B-ALL. A normal karyotype was detected. No fusion genes were detected using a multiplex RT-PCR panel covering 43 fusion genes commonly seen in acute leukemia (Supplementary Table 1). No mutations were detected using a targeted next-generation sequencing (NGS) panel covering 172 genes (Supplementary Table 2). The EP300ZNF384 fusion was detected firstly using RNA sequencing and further confirmed with Sanger sequencing (Supplementary Figure 1A), with fusion of exon 6 of EP300 to exon 3 of ZNF384. The patient received chemotherapy (mitoxantrone $10 \mathrm{mg}$ on day 1 , vincristine $4 \mathrm{mg}$ on day 1 , and dexamethasone $14 \mathrm{mg}$ on days $1-7$ ) but he still had $82 \%$ of blasts. Considering very weak expression of CD20 (0.13\%) and high expression of CD19 (98.3\%) and CD22 (95.2\%) (Figure 1B) in the blasts, he was enrolled in a phase I/II study involving tandem anti-CD19 and CD22 CAR-T cells in R/R B-ALL (NCT03614858). Chemotherapy (fludarabine $30 \mathrm{mg} / \mathrm{m}^{2}$ and cyclophosphamide $300 \mathrm{mg} / \mathrm{m}^{2}$ ) was administered 5, 4 and 3 days before the first infusion of CAR T-cells (June 27, 2020), which was followed by three infusions administered for the next 3 days. The effective antiCD19/CD22 CAR T-cells totaled $1 \times 10^{7} / \mathrm{kg}$ (Figure 1C). He developed high fever, hypotension and elevated IL-6. Grade 3 cytokine release syndrome (CRS) was determined according to ASTCT Consensus ${ }^{8}$ (Figure 1D). The CRS was relieved by dexamethasone, high-dose norepinephrine and symptomatic treatments. No signs of immune effector cell-associated neurotoxicity syndrome (ICANS) were observed. At 28 days post CAR T-cell therapy, the BM smear revealed no blasts, and flow cytometry analysis showed $4.3 \times 10^{-4}$ blasts (Table 1). Amplification of CAR T-cell as detected by qPCR peaked at day 12 and remained detectable before HSCT (Figure 1E). At the same time, he tested negative for the EP300-ZNF384 fusion. He underwent haploidentical allogeneic stem cell transplantation (alloHSCT) with his sister as donor one month after CAR T-cell therapy. BM evaluation at a month after HSCT showed $<2.4$ $\times 10^{-5}$ of blasts by flow cytometry and negative EP300ZNF384 with qPCR. Following HSCT, he exhibited grade IV acute graft-versus-host disease (GVHD) involving the intestine and liver according to the European Bone Marrow Transplantation consensus, ${ }^{9}$ which was relieved with tacrolimus, monoclonal antibody against CD25, etanercept, fecal microbiota transplantation and supportive care. At present, he has maintained continuous measurable residual disease (MRD)-negative remission $(<0.01 \%$ of bone marrow blasts by flow cytometry) for 14 months post HSCT.

Case 2 was a 25-year-old female diagnosed in January 2020 with B-ALL in the standard risk group. White blood count at diagnosis was $18.93 \times 10^{9} / \mathrm{L}$. CR was achieved after chemotherapy with idarubicin, vincristine, pegaspargase and prednisone. After consolidation chemotherapy with the hyper-CVAD regimen, hematologic relapse was detected in June 2020. BM morphology showed that $60 \%$ of blasts were positive for CD13, CD19, CD22, CD33, CD34, CD38, cCD79a, and HLA-DR and negative for CD20 (Figure 2A). A normal karyotype was detected. The results of multiplex PCR were negative. The EP300-ZNF384 fusion gene was also detected with RNA sequencing, with fusion of exon 6 of EP300 to exon 3 of ZNF384 (Supplementary Figure 1B). The NGS results showed KRAS Glu63_Ala66delinAspProThr and KMD6A Leu231fs mutations. After reinduction chemotherapy (idarubicin $10 \mathrm{mg}$ on day 1 , vincristine $4 \mathrm{mg}$ on day 1 , and dexamethasone $14 \mathrm{mg}$ on days 1-7), the blasts remained at $20.5 \%$. Due to the weak expression of $\mathrm{CD} 20(1.13 \%)$ and the high expression of CD19 $(100 \%)$ and CD22 (67.3\%), she was enrolled in the same clinical trial and received the same chemotherapy 5, 4 and 3 days before the first infusion of CAR T-cells, which was followed by three infusions administered for 
A

\begin{tabular}{l|l|l|l|l|l|l|l|}
\hline SP CD22 VL & CD19 VH & CD19 VL & CD22 VH & Hinge \&TM & CD28 \&OX40 & CD3ろ \\
\hline
\end{tabular}

B

before CAR T-cell Therapy
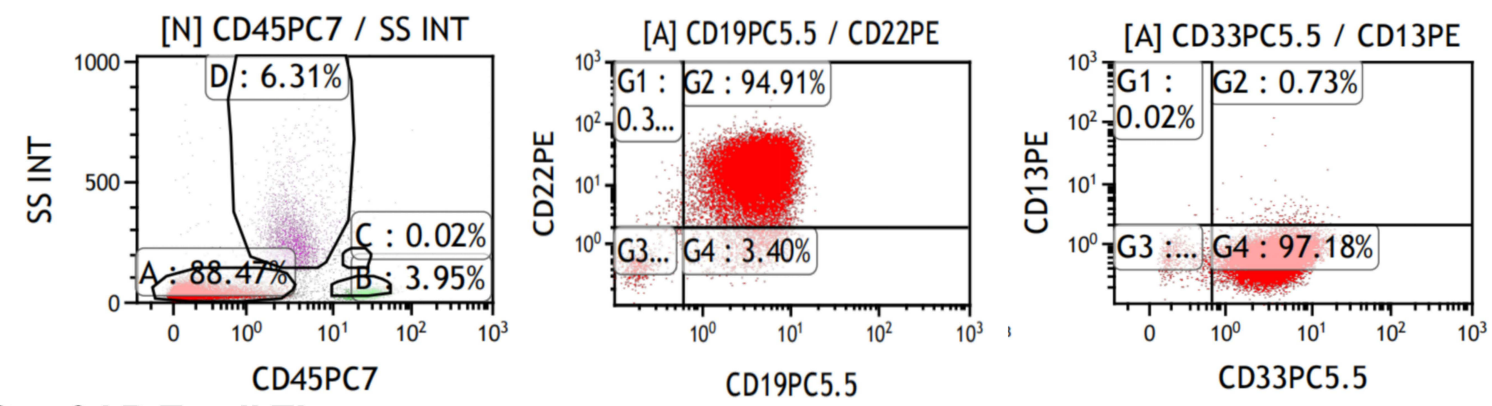

after CAR T-cell Therapy
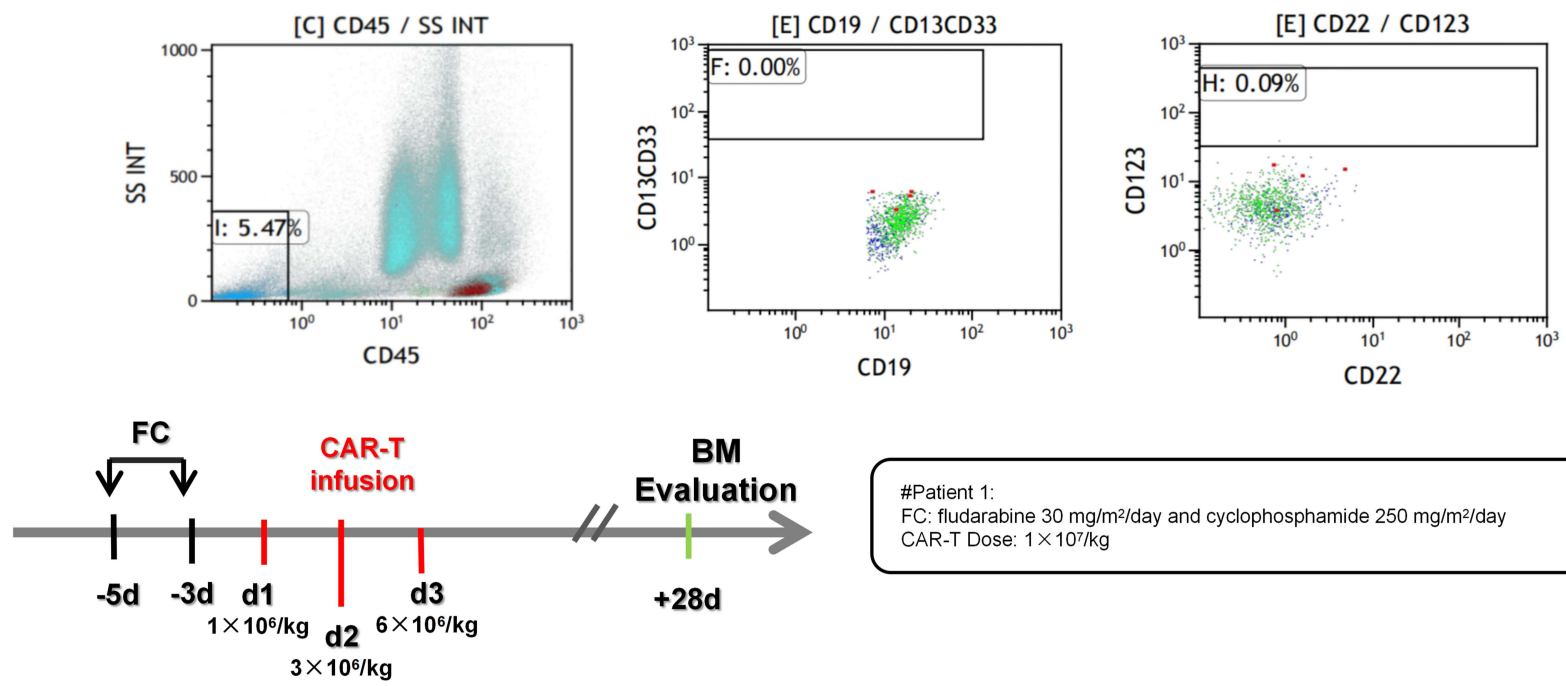

\#Patient 1:

FC: fludarabine $30 \mathrm{mg} / \mathrm{m}^{2} /$ day and cyclophosphamide $250 \mathrm{mg} / \mathrm{m}^{2} /$ day CAR-T Dose: $1 \times 10^{7} / \mathrm{kg}$

D

E
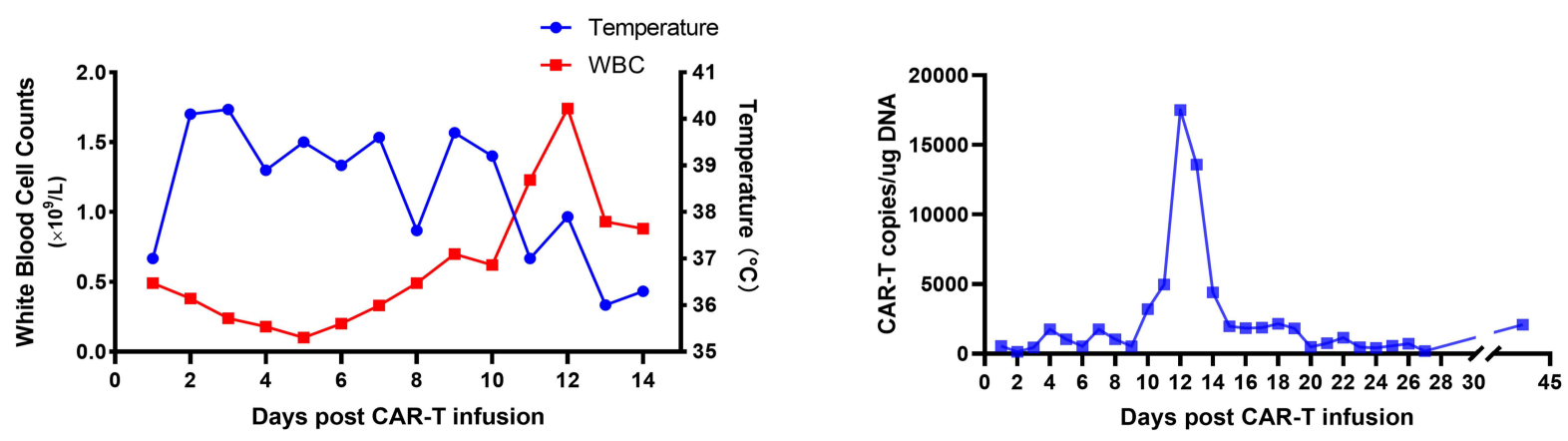

Figure I Clinical characteristics of Case I. (A) The structure of tandem CDI9/CD22 CAR. (B) Flow cytometry analysis of BM samples before and after CAR T-cell infusion. (C) The infusion schedule of Case I, with FC conditioning regimen and 3 consecutive days CAR T-cells infusion. (D) The changes in temperature and the WBC count post CAR T-cell infusion. Case I suffered high fever at day 3 after infusion. With the increase in the WBC count and supportive treatment, the patient's temperature returned to normal. (E) CAR T-cell copies detected by qPCR post infusion. 
Table I Clinical Characteristics of Patients at Relapse

\begin{tabular}{|c|c|c|c|}
\hline & & Case I & Case 2 \\
\hline \multicolumn{2}{|l|}{ Diagnosis time } & February 2020 & January 2020 \\
\hline \multicolumn{2}{|l|}{ Relapse time } & April 2020 & June 2020 \\
\hline \multirow[t]{9}{*}{ Baseline characteristics of both patients } & WBC $\left(\times 10^{9} / \mathrm{L}\right)$ & 4.56 & 18.93 \\
\hline & PLT $\left(\times 10^{9} / \mathrm{L}\right)$ & $|3|$ & 66 \\
\hline & $\mathrm{Hb}(\mathrm{g} / \mathrm{L})$ & 239 & 34 \\
\hline & BM blasts in morphology, \% & 82 & 20 \\
\hline & BM blasts in flow cytometry, \% & 88.47 & 20.50 \\
\hline & Karyotype & 46, $X Y[20]$ & 46, $X X[20]$ \\
\hline & Fusion gene by multiplex PCR & Negative & Negative \\
\hline & RNA-Sequencing & EP300-ZNF384 & EP300-ZNF384 \\
\hline & Mutations by targeted DNA-NGS & Negative & $\begin{array}{c}\text { KRAS Glu63_Ala66delin AspProThr I } \\
\text { KMD6A Leu23 Ifs }\end{array}$ \\
\hline \multirow[t]{6}{*}{ CAR T-cells therapy } & Dose of CAR T-cells infused & $1.0 \times 10^{7} / \mathrm{kg}$ & $2 \times 10^{7} / \mathrm{kg}$ \\
\hline & Grade of CRS & 3 & 2 \\
\hline & Grade of ICANS & No & No \\
\hline & BM blasts in morphology, \% & $0 \%$ & $0 \%$ \\
\hline & MRD by flow cytometry & $4.3 \times 10^{-4}$ & $<1 \times 10^{-4}$ \\
\hline & EP300-ZNF384 by qPCR & Negative & Negative \\
\hline \multirow[t]{6}{*}{ HSCT } & Donor Origin & Sister & Sister \\
\hline & HLA match & $5 / 10$ & $5 / 10$ \\
\hline & CD3+ count & $1.74 \times 10^{8} / \mathrm{kg}$ & $2.23 \times 10^{8} / \mathrm{kg}$ \\
\hline & CD34+ count & $3.26 \times 10^{6} / \mathrm{kg}$ & $8.62 \times 10^{6} / \mathrm{kg}$ \\
\hline & MNC count & $5.18 \times 10^{8} / \mathrm{kg}$ & $13.06 \times 10^{8} / \mathrm{kg}$ \\
\hline & GVHD & Grade IV aGVHD & No \\
\hline
\end{tabular}

Abbreviations: WBC, white blood cells; PLT, platelet; Hb, hemoglobin; BM, bone marrow; FCM, flow cytometry; PCR, polymerase chain reaction; NGS, next generation sequencing; CRS, cytokine release syndrome; ICANS, immune effector cell-associated neurotoxicity syndrome; CAR, chimeic antigen receptor; CR2, second complete remission; HSCT, hematopoietic stem cell transplantation; MNC, mononuclear cells; GVHD, graft versus host disease.

the next 3 days. The effective anti-CD19/CD22 CAR T-cells totaled $2 \times 10^{7} / \mathrm{kg}$ (Figure $2 \mathrm{~B}$ ). She suffered grade $2 \mathrm{CRS}$ and presented with fever and hypotension, which was relieved by symptomatic treatments and lowdose norepinephrine (Figure 2C). No symptoms of ICANS were observed. Amplification of CAR T-cell as detected by qPCR peaked at day 10 and dropped detectable before HSCT (Figure 2D). At 28 days post CAR T-cell therapy, BM evaluation showed $<1 \times 10^{-4}$ of blasts by flow cytometry and negative EP300-ZNF384 with qPCR, which demonstrated that MRD-negative CR was achieved (Table 1). In addition, results for the EP300-ZNF384 fusion and KRAS and KMD6A mutations all became negative. She underwent a haploidentical allo-HSCT with her sister as donor at a month post CAR T-cell therapy. No signs of GVHD were observed, and she remained in MRD-negative CR for 13 months after CAR-T therapy. 
A before CAR T-cell Therapy


\section{after CAR T-cell Therapy}


B



\#Patient 2:

FC: fludarabine $30 \mathrm{mg} / \mathrm{m}^{2} /$ day and cyclophosphamide $250 \mathrm{mg} / \mathrm{m}^{2} /$ day CAR-T Dose: $2 \times 10^{7} / \mathrm{kg}$

C



D



Figure 2 Clinical characteristics of Case 2. (A) Flow cytometry analysis of BM samples before and after CAR T-cell infusion. (B) The infusion schedule of case 2. The patient got 4 days CAR T-cells infusion with $2 \times 10^{7} / \mathrm{kg}$. (C) The changes in temperature and the WBC count post CAR T-cell infusion. Case 2 suffered high fever at day 4 after infusion. With the increase in the WBC count and supportive treatment, the patient's temperature returned to normal. (D) CAR T-cell copies detected by qPCR post infusion.

\section{Discussion}

EP300-ZNF384-positive B-ALL is a rare subtype with a characteristic immunophenotype and is more prevalent in adolescents and young adults according to the limited number of cases reported thus far. ${ }^{3,4}$ Both patients presented here were young adults, showing abnormal expression of CD13 and CD33 and weak expression of CD20 at relapse, in accordance with previous studies. ${ }^{3}$ Both EP300 and ZNF384 are located 
within close proximity of the telomeres of their respective chromosomes, which makes EP300-ZNF384 difficult to identify by conventional cytogenetic analysis. Therefore, patients with R/R B-ALL and a normal karyotype should be screened for EP300-ZNF384 using transcriptome sequencing. EP300ZNF384 is a newly identified fusion gene in B-ALL, with only 53 cases reported hitherto (Table 2). Most patients harboring the EP300-ZNF384 fusion achieved CR and had $62.5 \%$ survival with conventional chemotherapy in a previous study. ${ }^{3,10,11}$ However, some patients with EP300-ZNF384 also suffered relapses and were refractory to salvage chemotherapy (Supplementary Table 3). ${ }^{5}$ Both patients reported here had weak expression of $\mathrm{CD} 20$ and therefore presented no target for anti-CD20 monoclonal antibody or CD20-targeted CAR T-cell therapy. As reported in most cases, exon 6 of EP300 was fused with exon 3 of ZNF384 in both cases. ${ }^{11}$ The EP300ZNF384 fusion protein lacks the histone acetyltransferase (HAT) domain of EP300, and retained the complete ZNF384 protein. ${ }^{11}$ EP300-ZNF384 without the HAT domains may be involved in the development of ALL by deregulating acetylation-mediated inactivation of the BCL6 oncoprotein and activation of the 53 tumor suppressor. ${ }^{13}$ In addition, the upregulation of the JAK-STAT pathway and reduced DNA repair capacity have also been identified in patients with EP300-ZNF384. ${ }^{3}$ However, there have been no in vitro or in vivo studies on the application of histone acetyltransferase regulators, JAK inhibitors or drugs targeting DNA repair pathways in this subtype.

CAR T-cell therapy has shown unprecedented success in R/R B-ALL. ${ }^{6}$ At present, only two EP300-ZNF384positive B-ALL patients have undergone CAR T-cell therapy; however, both patients died. ${ }^{5}$ No details about the efficacy and safety of CAR T-cells for these patients were reported. Despite the high response rate of CD19 CAR T-cell therapy, approximately $50 \%$ of R/R B-ALL patients relapsed after CD19 CAR T-cell therapy, which is partially due to CD19 antigen loss. ${ }^{6}$ CD19 and CD22 dual-targeted CAR T-cells have demonstrated potential in reducing the relapse rate of $\mathrm{R} / \mathrm{R}$ B-ALL patients with antigen loss in clinical trials. ${ }^{14}$ Because of the concurrently high

Table 2 Summary of Genetic Details of Patients with EP300-ZNF384

\begin{tabular}{|c|c|c|c|c|c|c|c|c|}
\hline \multirow[t]{2}{*}{ No } & \multirow[t]{2}{*}{ Author } & \multirow[t]{2}{*}{ Year } & \multirow{2}{*}{$\begin{array}{c}\text { No. of } \\
\text { Patients }\end{array}$} & Age & \multirow[t]{2}{*}{ Sex } & \multirow[t]{2}{*}{ Methods } & \multirow[t]{2}{*}{ Clinical Response } & \multirow{2}{*}{$\begin{array}{l}\text { Last } \\
\text { Status }\end{array}$} \\
\hline & & & & $\begin{array}{l}\text { Median } \\
\text { (Range) }\end{array}$ & & & & \\
\hline I & Gocho et al ${ }^{\prime \prime}$ & 2015 & 6 & $11.5(7 \sim 17)$ & $4 \mathrm{M} 2 \mathrm{~F}$ & RNA-seq & $6 \mathrm{CR}$ & 6 Alive \\
\hline 2 & Griffith et al ${ }^{12}$ & 2016 & I & 25 & M & $\begin{array}{c}\text { WGS, WES, RNA- } \\
\text { seq, }\end{array}$ & Relapse & Dead \\
\hline 3 & Liu et $\mathrm{al}^{16}$ & 2016 & 14 & $19(4.1 \sim 56.3)$ & Uk & $\begin{array}{c}\text { WGS, WES, RNA- } \\
\text { seq, }\end{array}$ & Unknown & $\begin{array}{l}\text { I Relapse } \\
2 \text { Death }\end{array}$ \\
\hline 4 & Shago et $\mathrm{al}^{10}$ & 2016 & I & 15 & $M$ & RNA-seq, & MRD+CR 9 years & Alive \\
\hline \multirow[t]{2}{*}{5} & \multirow{2}{*}{$\begin{array}{l}\text { Hirabayashi } \\
\quad \text { et al }\end{array}$} & \multirow[t]{2}{*}{2017} & \multirow[t]{2}{*}{9} & \multirow[t]{2}{*}{ Uk } & \multirow[t]{2}{*}{$6 \mathrm{M} 3 \mathrm{~F}$} & \multirow[t]{2}{*}{ RNA-seq, } & \multirow[t]{2}{*}{$8 \mathrm{CR}$ I NR } & $\begin{array}{l}\text { 5y LFS: } \\
83.3 \%\end{array}$ \\
\hline & & & & & & & & $\begin{array}{l}5 y \text { OS: } \\
100 \%\end{array}$ \\
\hline 6 & McClure et al ${ }^{3}$ & 2018 & 9 & $24.5(4.4 \sim 47)$ & $7 \mathrm{M} 2 \mathrm{~F}$ & RNA-seq, & 6 CRI 3Relapse & $\begin{array}{l}4 \text { Alive } \\
5 \text { Death }\end{array}$ \\
\hline 7 & Jing et $\mathrm{al}^{5}$ & 2020 & 10 & $30(17 \sim 63)$ & $5 \mathrm{M} 5 \mathrm{~F}$ & RNA-seq & $7 \mathrm{CR} 3 \mathrm{NR}$ & $\begin{array}{l}7 \text { Alive } \\
3 \text { Death }\end{array}$ \\
\hline \multirow[b]{2}{*}{8} & \multirow[b]{2}{*}{ Yao et $\mathrm{al}^{17}$} & \multirow[b]{2}{*}{2020} & \multirow[b]{2}{*}{3} & \multirow[b]{2}{*}{$26(23 \sim 27)$} & \multirow[b]{2}{*}{$2 M I F$} & \multirow[b]{2}{*}{ RNA-seq } & 2 CRI(Chem-HSCT) & \multirow[b]{2}{*}{$\begin{array}{l}2 \text { Alive } \\
\text { I Death }\end{array}$} \\
\hline & & & & & & & $\begin{array}{c}\text { I CR3 (Chem-CAR- } \\
\text { HSCT) }\end{array}$ & \\
\hline
\end{tabular}

Abbreviations: M, male; F, female; WGS, whole genome sequencing; WES, whole exome sequencing; uk, unknown; LFS, leukemia free survival; OS, overall survival; MRD $+C R$, minimal residual disease positive complete remission; NR, no remission; Chem, chemotherapy; CAR, chimeric antigen receptor; HSCT, hematopoietic stem cell transplantation. 
expression of CD19 and CD22, we applied tandem CD19/ CD22 CAR T-cell therapy in both patients to avoid relapse. Both patients reported here were chemo-resistant and had high tumor burden before infusion of CAR T cells. At day 28, in the BM evaluation, both patients achieved $\mathrm{CR}$, which demonstrated the potent antileukemic activity of the CD19/CD22 CAR T-cells. No symptoms of ICANS were observed. All toxicities related to CRS were relieved with symptomatic treatments and supportive care. Hence, this bispecific CD19/CD22 CAR T-cell infusion appears safe and effective in EP300-ZNF384-positive patients with a high tumor burden. With CAR T-cell therapy bridging to allo-HSCT, the 1-year overall survival and event-free survival for R/R B-ALL were shown to increase to $87.7 \%$ and $73 \%$, respectively in a large cohort. ${ }^{15}$ Therefore, alloHSCT was subsequently performed in both patients to prevent relapse and achieve sustainable remission. Both patients had maintained $\mathrm{CR}$ at the last follow-up.

To our knowledge, this is the first report of EP300 ZNF384-positive B-ALL patients achieving continuous deep remission through tandem CD19/CD22 CAR T-cell therapy bridging to allo-HSCT. Due to the lower incidence of EP300-ZNF384-positive R/R B-ALL and the limited number of patients in this study, the outcome of such a strategy in EP300-ZNF384-positive B-ALL needs to be investigated in a larger, multiple-center clinical trial.

\section{Ethical Approval and Consent}

This study was approved by the Ethics Committee of the First Affiliated Hospital of Soochow University and was conducted in accordance with the principles of the Declaration of Helsinki. Both patients provided written informed consent, and they consented to the publication of their clinical details.

\section{Acknowledgements}

This work was supported by research grants from National Natural Science Foundation of China (81873443, 82070162), the Frontier Clinical Technical Project of the Science and Technology Department of Jiangsu Province (BE2017655), Jiangsu Provincial Medical Talent (ZDRCA2016045), Major Natural Science Research Projects in institutions of higher education of Jiangsu Province (19KJA210002), The Key Science Research Project of Jiangsu Commission of Health (K2019022), Translational Research Grant of NCRCH (2020ZKZC04) and Natural Science Foundation of Jiangsu Province (BK20190181, BK20201169), the Priority Academic
Program Development of Jiangsu Higher Education Institutions (PAPD).

\section{Disclosure}

The authors report no conflicts of interest in this work.

\section{References}

1. Malard F, Mohty M. Acute lymphoblastic leukaemia. Lancet. 2020;395(10230):1146-1162. doi:10.1016/S0140-6736(19)33018-1

2. Li JF, Dai YT, Lilljebjörn H, et al. Transcriptional landscape of B cell precursor acute lymphoblastic leukemia based on an international study of 1223 cases. Proc Natl Acad Sci U S A. 2018;115(50): E11711-e11720. doi:10.1073/pnas.1814397115

3. McClure BJ, Heatley SL, Kok CH, et al. Pre-B acute lymphoblastic leukaemia recurrent fusion, EP300-ZNF384, is associated with a distinct gene expression. Br J Cancer. 2018;118(7):1000-1004. doi:10.1038/s41416-018-0022-0

4. Hirabayashi S, Ohki K, Nakabayashi K, et al. ZNF384-related fusion genes define a subgroup of childhood B-cell precursor acute lymphoblastic leukemia with a characteristic immunotype. Haematologica. 2017;102(1):118-129. doi:10.3324/haematol.2016.151035

5. Jing Y, Li YF, Wan H, Liu DH. Detection of EP300-ZNF384 fusion in patients with acute lymphoblastic leukemia using RNA fusion gene panel sequencing. Ann Hematol. 2020;99(11):2611-2617. doi:10.1007/s00277-020-04251-8

6. Jackson HJ, Rafiq S, Brentjens RJ. Driving CAR T-cells forward. Nat Rev Clin Oncol. 2016;13(6):370-383. doi:10.1038/nrclinonc.2016.36

7. Tanguy-Schmidt A, Rousselot $\mathrm{P}$, Chalandon $\mathrm{Y}$, et al. Long-term follow-up of the imatinib GRAAPH-2003 study in newly diagnosed patients with de novo Philadelphia chromosome-positive acute lymphoblastic leukemia: a GRAALL Study. Biol Blood Marrow Transplant. 2013;19(1):150-155. doi:10.1016/j.bbmt.2012.08.021

8. Lee DW, Santomasso BD, Locke FL, et al. ASTCT consensus grading for cytokine release syndrome and neurologic toxicity associated with immune effector cells. Biol Blood Marrow Transplant. 2019;25 (4):625-638. doi:10.1016/j.bbmt.2018.12.758

9. Przepiorka D, Weisdorf D, Martin P, et al. 1994 Consensus conference on acute GVHD grading. Bone Marrow Transplant. 1995;15 (6):825-828.

10. Shago M, Abla O, Hitzler J, Weitzman S, Abdelhaleem M. Frequency and outcome of pediatric acute lymphoblastic leukemia with ZNF384 gene rearrangements including a novel translocation resulting in an ARID1B/ZNF384 gene fusion. Pediatr Blood Cancer. 2016;63 (11):1915-1921. doi:10.1002/pbc.26116

11. Gocho $\mathrm{Y}$, Kiyokawa $\mathrm{N}$, Ichikawa $\mathrm{H}$, et al. A novel recurrent EP300-ZNF384 gene fusion in B-cell precursor acute lymphoblastic leukemia. Leukemia. 2015;29(12):2445-2448. doi:10.1038/leu.2015.111

12. Griffith M, Griffith OL, Krysiak K, et al. Comprehensive genomic analysis reveals FLT3 activation and a therapeutic strategy for a patient with relapsed adult B-lymphoblastic leukemia. Exp Hematol. 2016;44(7):603-613. doi:10.1016/j.exphem.2016.04.011

13. Pasqualucci L, Dominguez-Sola D, Chiarenza A, et al. Inactivating mutations of acetyltransferase genes in B-cell lymphoma. Nature. 2011;471(7337):189-195. doi:10.1038/nature09730

14. Pan J, Zuo S, Deng B, et al. Sequential CD19-22 CAR T therapy induces sustained remission in children with $\mathrm{r} / \mathrm{r}$ B-ALL. Blood. 2020;135(5):387-391. doi:10.1182/blood.2019003293

15. Zhang Y, Chen H, Song Y, et al. Chimeric antigens receptor T cell therapy as a bridge to haematopoietic stem cell transplantation for refractory/ relapsed B-cell acute lymphomalastic leukemia. $\mathrm{Br}$ J Haematol. 2020;189(1):146-152. doi:10.1111/bjh.16339 
16. Liu YF, Wang BY, Zhang WN, et al. Genomic profiling of adult and pediatric B-cell acute lymphoblastic leukemia. EBioMedicine. 2016;8:173-183. doi:10.1016/j.ebiom.2016.04.038
17. Yao ZL, Li YF, Li M, et al. Analysis of clinical characteristics of acute B lymphoblastic leukemia with EP300-ZNF384 fusion gene positive. Zhongguo Shi Yan Xue Ye Xue Za Zhi. 2020;28(1):24-28.

\section{Publish your work in this journal}

OncoTargets and Therapy is an international, peer-reviewed, open access journal focusing on the pathological basis of all cancers, potential targets for therapy and treatment protocols employed to improve the management of cancer patients. The journal also focuses on the impact of management programs and new therapeutic

Submit your manuscript here: https://www.dovepress.com/oncotargets-and-therapy-journal agents and protocols on patient perspectives such as quality of life, adherence and satisfaction. The manuscript management system is completely online and includes a very quick and fair peer-review system, which is all easy to use. Visit http://www.dovepress.com/ testimonials.php to read real quotes from published authors. 